\title{
Correlation of microvessel density with tumor type, tumor size, histological grade and lymph node status of breast carcinoma
}

\author{
V. Vijayasree', S. V Ramana ${ }^{2, *}$, R.S. Ashok Kumar ${ }^{3}$, Ranveer Singh ${ }^{4}$, Dr. Sandhya Anil ${ }^{5}$ \\ ${ }^{1,2}$ Associate Professor, ${ }^{3-5}$ Professor, Dept. of Pathology, Kamineni Institute of Medical Sciences, Narketpally \\ Telangana, ${ }^{2}$ Vishwabharathi Medical College, Kurnool, Andhra Pradesh, ${ }^{3}$ MNR Medical College, Telangana, ${ }^{4}$ Medicity Medical \\ College, Medchal, Telangana, ${ }^{5}$ Kakatiya Medical College, Telangana, India
}

\author{
*Corresponding Author: \\ Email: vijayasree_vennila@yahoo.co.in
}

Received: $1^{\text {st }}$ September, 2017

Accepted: $18^{\text {th }}$ October, 2017

\begin{abstract}
Introduction: Microvessel density is used in assessment of the angiogenesis, a prognostic factor for many malignancies. While it has extensively been studied in cancers of lung, brain, colon, prostate as well as in melanoma, but, there are scanty reports for the study of microvessel density of breast carcinoma.

Aim: Present study was aimed to find the correlation of microvessel density with tumor type, tumor size, histological grade and lymph node status.

Study Design: The biopsy specimens, from 38 women diagnosed for carcinoma breast who underwent modified radical mastectomy and subsequently were examined with routine H\&E stain and immunoperoxidase for CD-31 marker. Micro vessel density was measured as micro vessel count $/ \mathrm{mm} 2$ on all the specimens of breast carcinoma. Micro vessel density correlated well with tumor size, tumor type, histological grade and lymph node status.

Results: micro vessel density had significant positive correlation with tumor type, histological grade, and lymph node status $(\mathrm{p}<0.05)$.
\end{abstract}

Conclusion: Estimation of micro vessel density could be used as the prognostic marker for breast carcinoma.

Keywords: Carcinoma of breast, Micro-vessel density (MVD), Histological grade, Lymph node status, Tumor type and tumor stage.

\section{Introduction}

Angiogenesis is a process in which preexisting vessels send out capillary buds or sprouts to produce new vessels or in other occasions, endothelial cells are recruited from the bone marrow and forms new blood vessel. $^{1}$

Tumor angiogenesis is the induction of the growth of blood vessels from surrounding tissue into a tumor by a diffusible protein factor released by the tumor cells. $^{2-4}$ Angiogenesis is an important process in healing and is critical to chronic inflammation, fibrosis as well as for tumor cell growth and to the formation of collateral circulation. ${ }^{1}$ It has extensively been studied in many malignancies like melanoma, lung, brain, colon and prostate, but, there are scanty reports for the study of angiogenesis in breast carcinoma.

Tumor cannot enlarge beyond one to two $\mathrm{mm}$ in diameter or thickness unless they are vascularized. Presumably this $1-2 \mathrm{~mm}$ zone represents the maximal distance across which oxygen and nutrients can diffuse from blood vessels to the tumor. Beyond this size, the tumor fails to enlarge without vascularization, and subsequently undergo apoptotic changes due to hypoxia. ${ }^{5}$ Neovascularization has a dual effect on the tumor growth. First of all, perfusion supplies nutrients and oxygen and then the newly formed endothelial cells stimulate the growth of adjacent tumor cells by secreting polypeptides such as, Insulin-like growth factors, PDGF, granulocyte-macrophage colony stimulating factor and interleukin. Angiogenesis is a prerequisite not only for continued tumor growth, but also for metastasis. ${ }^{6,7}$

Several studies have revealed a correlation between the extent of angiogenesis and the probability of the metastasis especially in melanomas and cancers of the breast, lung, colon and prostate. In some of these cancers especially breast and prostate, vessel density has proven to be a significant prognostic indicator. ${ }^{8}$

In our study, we have done microvessel quantitation in different types of breast carcinomas by adopting staining method by Colette charpin et al and Horak et al i.e.CD-31 immunoperoxidase staining of endothelial cells. ${ }^{10,11}$

\section{Materials and Methods}

Breast tumor specimens were obtained from 38 patients who presented to the department of surgery, Kakathiya medical college, NTRUHS, Andhra Pradesh, India. These patients underwent modified radical mastectomy.

The tumor specimens were received from operating room and fixed in 10\% buffered formalin. Tissue sections were prepared, and stained with hematoxylineosin for histological assessment. Tumor grades and typing were assessed on H\&E-stained histological sections. The tumor grading was done according to 
"modified blooms Richardson criteria". 9 Multiple sections of breast tumor were chosen from the routine $\mathrm{H}$ \& $\mathrm{E}$ slides for this purpose. One representative section per case was used for microvessel staining.

Microvessel staining was done by using CD-31 immunohistochemical stain for visualizing endothelial cells by immunoperoxidase method. Those slides were counter stained with Haematoxylin. ${ }^{10,11}$

The microscope used for micro vessel analysis was Germany-Leitz wetzlar light microscope. The sections were studied for microvessel density, at low magnification (10 x objective, 10 ocular; area $=1.5129$ sq.mm) and vessels were counted randomly on twenty $400 \mathrm{x}$ fields ( $40 \mathrm{x}$ objective, with a $10 \mathrm{x}$ ocular) under ocular grid which was having $0.1024 \mathrm{~mm}^{2}$ area. Out of those twenty fields, three highly vascular fields were selected and recorded. The mean values of these three highly vascular fields were calculated to ensure reproducibility. Those areas of high vascularization occured anywhere in the invasive tumor but were most frequently seen at the margins of the carcinoma.

Micro vessels in sclerotic areas within the tumors, where the micro vessels were sparse, and microvessels in immediately adjacent areas of tumor that was unaffected breast tissue were not considered in vessels counts. However these micro vessels served as internal controls.
We standardized, the vascularisation as the number of vessels per squire millimeters calculated from readings of vessels per $0.1024 \mathrm{~mm}^{2}$ under $400 \mathrm{x}$. This standard method was suggested by Weidner et al and also used by many other workers on angiogenesis in various carcinomas. ${ }^{12}$

Because of higher resolution of the $40 \mathrm{x}$ objective more vessels were found, therefore $40 \mathrm{X}$ objective were used for vessel counting.

We used following criteria for micro vessels counting. ${ }^{12,13}$

Besides counting the fully developed microvessels, we also considered counting those newly forming single endothelial cells or clusters of endothelial cells with or without lumen as single a vessel.

Vessels of a caliber larger than approximately eight red blood cells, vessels with thick muscular walls, and vessels in sclerotic areas were excluded from counting.

Micro vessel counts and density were performed blindly without previous knowledge of the presence or absence of metastasis and the histological grade.

\section{Results}

38 cases of invasive carcinoma of breast were studied and the range of age of those cases was 28 years to 81 years, with a mean age of 50.3 . (Graph 1 )

Graph 1: percentage of age distribution of invasive breast carcinoma

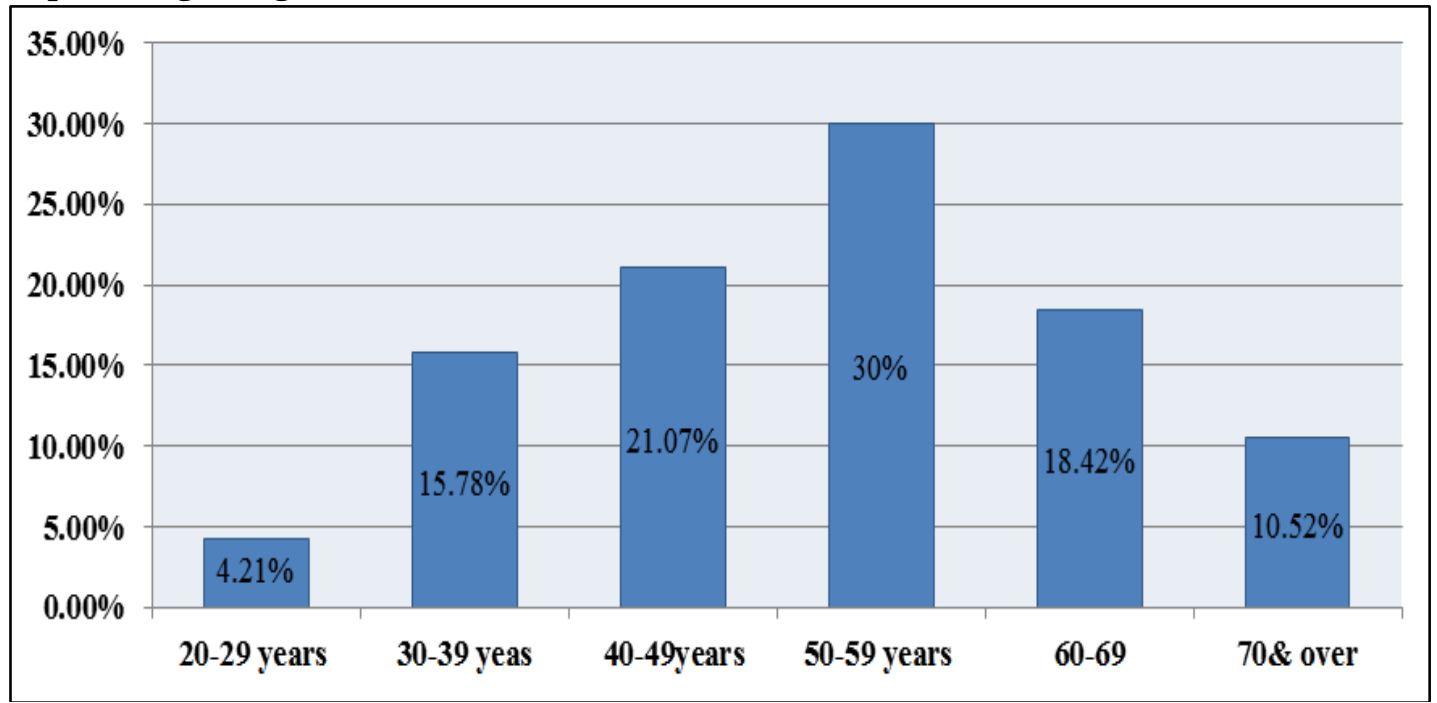

From the $\mathrm{H} \& \mathrm{E}$ stain, 38 breast carcinoma cases were segregated into 7types, those were 1. Ductal Carcinoma- No special type -19 cases $(50.81 \%), 2$. Medullary carcinoma-6 cases (15\%); 3 . No special type with occasional comedo pattern-5cases $(13.15 \%) ; 4$. Ductal carcinoma with majority comedo pattern- 4 cases (10.52\%), 5. Papillary carcinoma- 1 case $(2.63 \%) ; 6$. Tubular carcinoma- 1 case $(2.63 \%)$, and 7 . lobular invasive carcinoma -2 cases $(5.26 \%)$. (Graph 2$)$. 
Graph 2: Tumor type distribution of invasive breast carcinoma

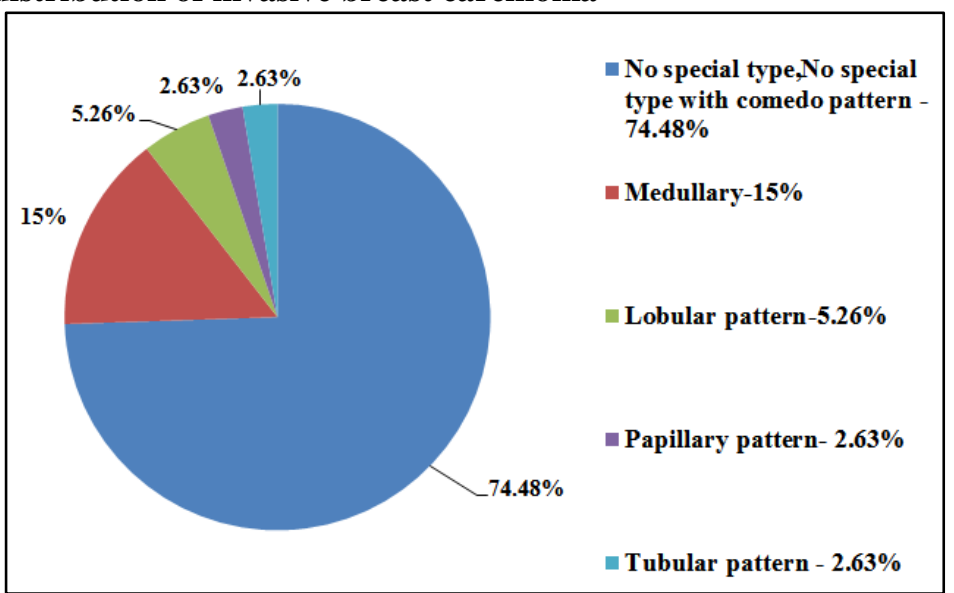

Histological grading was done according to 'Nottingham modification of Bloom - Richardson' system. The 19 'Ductal carcinoma NST' showed $8(42.1 \%)$ cases of the Grade $-\mathrm{I}, 9$ cases $(47.36 \%)$ Grade-II, and 2 cases (10.52\%) Grade- III. 5 of the
'NST with comedo pattern' showed 4 cases (80\%) of Grade-II and 1 case (20\%) of Grade -III, and 4out of 4 comedo pattern cases $(100 \%)$ of Ductal carcinoma with grade- II. (Graph 3)

\section{Graph 3: Histological Grade}

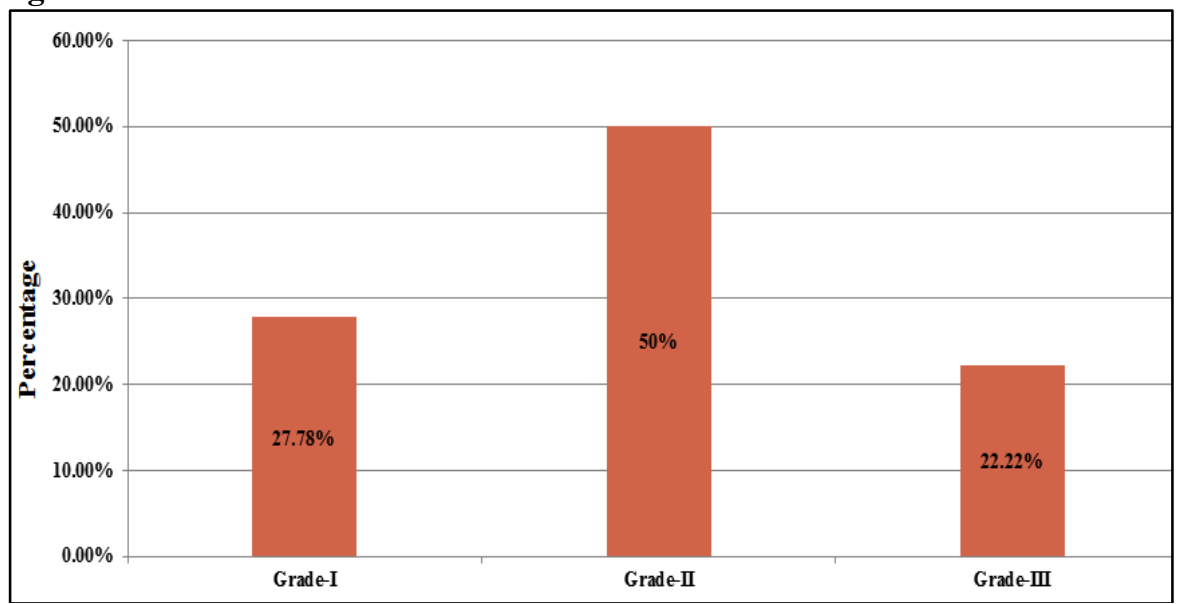

Of, all these 38 cases, 13 cases $(32.57 \%)$ were associated with stage 1 lymph node status, 16 cases
$(43.75 \%)$ with stage-2 and 9 cases $(23.68 \%)$ with stage -3 lymph nodal status. (Graph 4)

\section{Graph 4: Lymph node status}

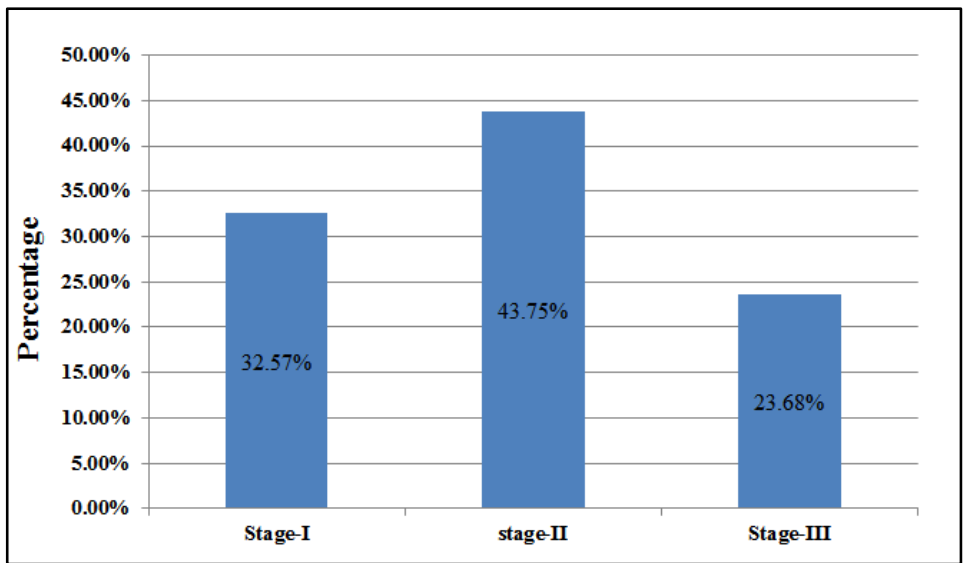


The lymph nodal status were as follows. Of the 8 cases of grade I NST, 4 showed lymph nodal status-1. 2 cases lymph nodal status 2 , and 2 cases lymph nodal status 3.

Of the 9cases of grade-II of NST, 3 showed lymph nodal status $-1,6$ showed lymph nodal status-2. Two cases of grade -III NST only showed lymph nodal status 2.

In 'No special type with comedo carcinoma included 5 cases. Of these 4 cases of grade-II, which again had 2 cases lymph nodal status 2, and another 2 cases lymph nodal status 3 . The other grade-III showed lymph nodal status-3.

In "Comedo pattern" series, among the 4 Grade- II cases- Three cases were associated with stage- 1 lymph node status and 1 case was stage- 3 lymph node status.

Of the 6 cases with medullary carcinoma, 5 cases were associated with stage -2 lymph node status and one case with stage 3 lymph node status.

\section{Graph 5: Microvessel density in different tumor types of breast carcinoma}

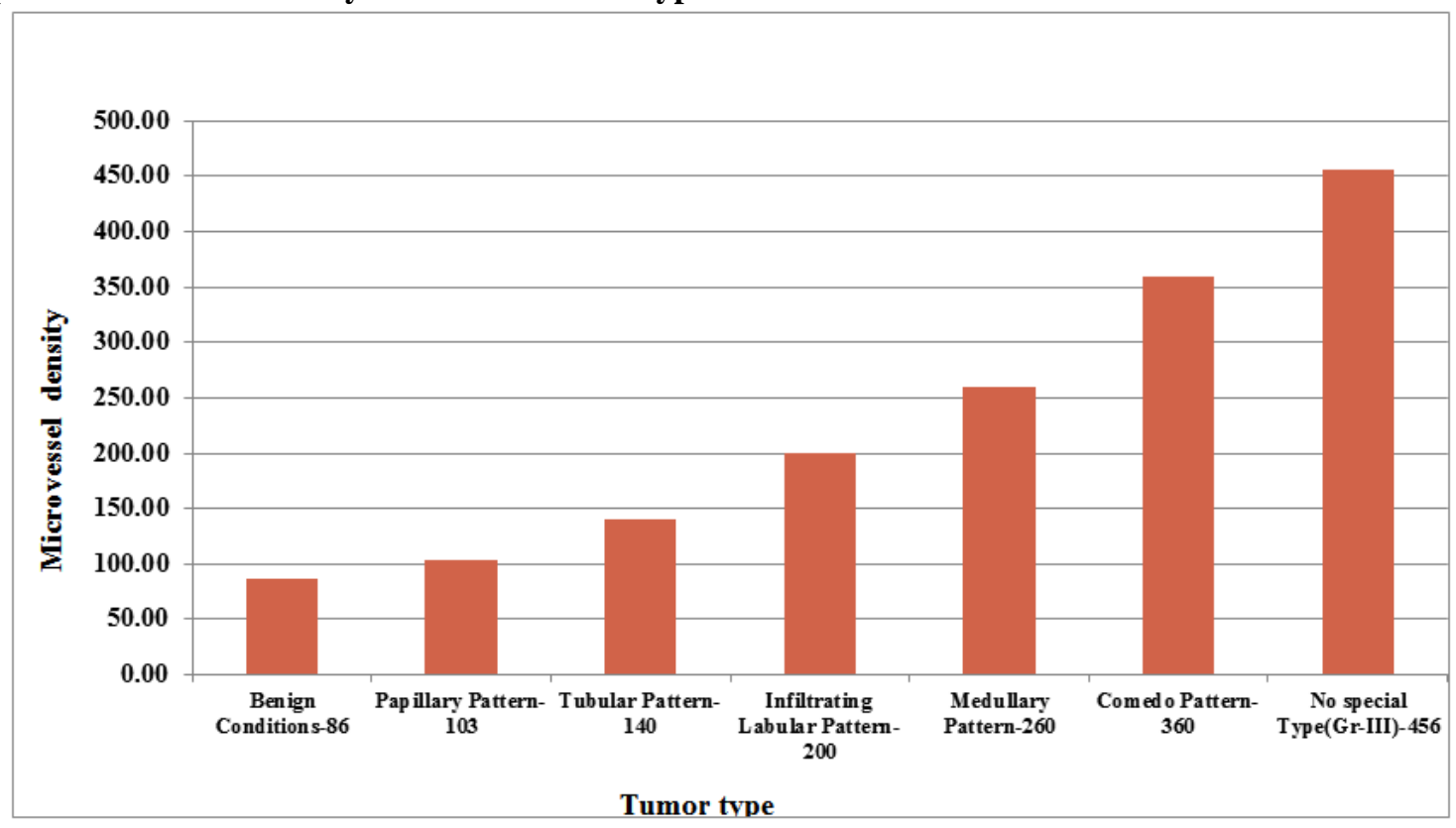

The only case of tubular carcinoma was associated with stage- 1 and one case of papillary carcinoma with stage-2 lymph node status. Two of the 'Invasive lobular carcinomas were associated with stage-1 lymph node status.

Microvessel density were identified as follows (Graph 5): 'No special type' invasive carcinoma with grade-I, II and III showed-176, 196, and 456 Microvessel count $/ \mathrm{mm}^{2}$ respectively. (Fig. 1)

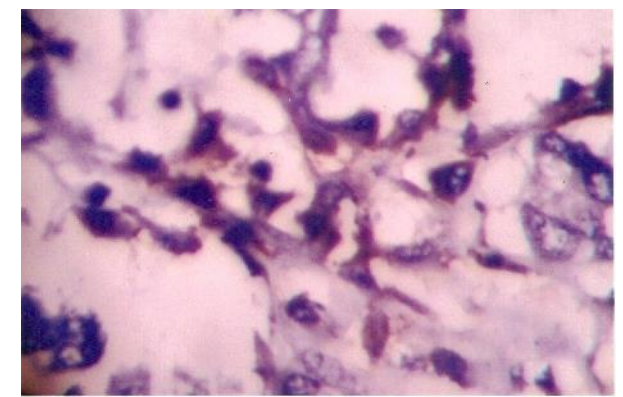

Fig. 1: Photomicrograph of the CD-31

immunoperoxidase stain, $\mathrm{X} 400$ No special type of infiltrating duct cell carcinoma, demonstrating vascularity
Comedo pattern showed 360 microvessel count $/ \mathrm{mm}^{2}$ and medullary carcinoma showed260 Microvessel count $/ \mathrm{mm}^{2}$. (Fig. 2).

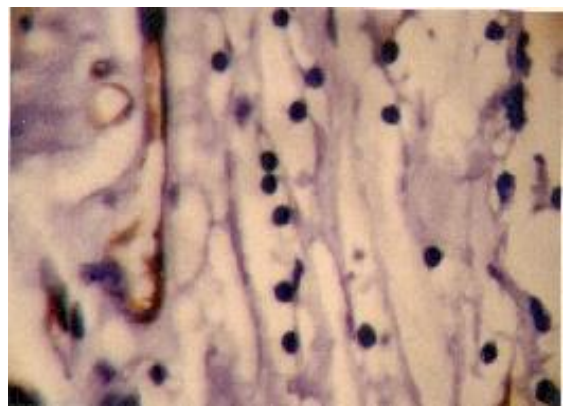

Fig. 2: Photomicrograph of the CD-31 immunoperoxidase stain, X400, medullary carcinoma

Papillary carcinoma showed 136 microvessel count $/ \mathrm{mm}^{2}$ and tubular pattern showed 146 microvessel count $/ \mathrm{mm}^{2}$. (Fig. 3) 


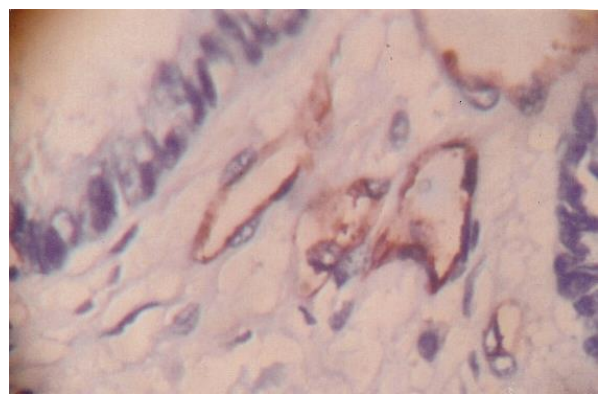

Fig. 3: PMG of the CD-31 immunoperoxidase stain, $\mathrm{X} 400$, papillary carcinoma demonstrating vascularity in the core of papilla

Infiltrating lobular carcinoma showed -200 Microvessel count $/ \mathrm{mm}^{2}$. (Fig. 4)

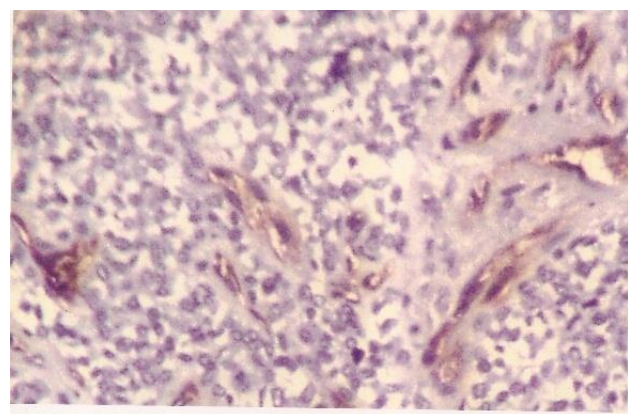

Fig. 4: PMG of the CD-31 immunoperoxidase stain, $\mathrm{X} 400$, lobular carcinoma

Fibrocystic diseases showed 86 microvessel count $/ \mathrm{mm}^{2}$ and Fibro adenoma showed 80 microvessel count $/ \mathrm{mm}^{2}$. (Fig. 5)

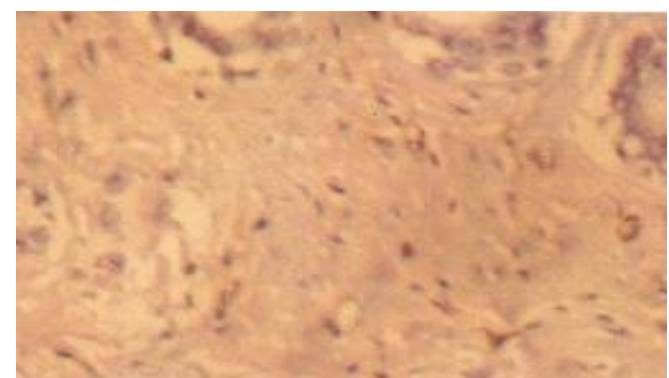

Fig. 5: PMG of the CD-31 immunoperoxidase, $X 400$, Fibroadenoma
Microvessel density correlation with lymph nodal status, histological grade, tumor type and tumor size was done as follows:

Among 'No special type' cases Grade-1 with stage1 lymph node status showed 193, stage- 2 showed 226 and stage -3 showed 236 microvessel count $/ \mathrm{mm}^{2}$. Grade -II with stage 1 showed 216, Grade II with stage-2 lymph node status showed 276, and Grade -III with stage -1 showed 350 and Grade III with stage-2 lymph node status showed - 413 microvessel count $/ \mathrm{mm}^{2}$.

Among 'No special type with comedo pattern' cases, Grade -II with stage -1 showed $250 \mathrm{MVC} / \mathrm{mm} 2$ and grade II with stage -3 lymph node status showed $263 \mathrm{MVC} / \mathrm{mm}^{2}$ and Grade III with stage 3 lymph node status showed 336 microvessel count $/ \mathrm{mm}^{2}$.

Among 'medullary pattern' cases stage -2 \&stage -3 lymph node status showed 243 and 260 microvessel count $/ \mathrm{mm}^{2}$ respectively.

Association between microvessel density and traditional prognostic indicators such as tumor size, histological grade, tubular differentiation, nuclear and mitotic grading as well as the lymph node status were evaluated by calculating 'pearson's coefficient' of correlation. Statistical analysis was performed using the "SPSS 11.5 version computer".

We found proportionally significant association between 'microvessel density' with tubular differentiation ('p' value $<0.05$ ), Nuclear grade ('p' value $<0.05$ ), Mitotic grade ('p' value $<0.05$ ), and Histological Grade ('p' value $<0.05$ ) of 'Nottingham modification of Bloom- Richardson grading system' and the lymph node status ('p' value $<0.05$ ) as well. There was no significant association present between tumor size and microvessel density (' $\mathrm{P}$ ' value $>0.05$ ). (Table 1)

Table 1: Correlation of micro vessels density with nottingham prognostic index, lymph node status and Tumor size

\begin{tabular}{|l|c|c|}
\hline Prognostic Factor & Highest Count ('p' $\mathbf{p}$ 'Value) & Mean Count ('p' value) \\
\hline 1 Histological grade & $<0.05(\mathrm{~S})$ & $<0.05(\mathrm{~S})$ \\
\hline A Tubule formation & $<0.05(\mathrm{~S})$ & $<0.01(\mathrm{~S})$ \\
\hline B Nuclear grade & $<0.05(\mathrm{~S})$ & $<0.05(\mathrm{~S})$ \\
\hline C Mitotic grade & $<0.05(\mathrm{~S})$ & $<0.05(\mathrm{~S})$ \\
\hline II Lymphnode status & $<0.05(\mathrm{~S})$ & $<0.05(\mathrm{~S})$ \\
\hline III Tumor size & $>0.05(\mathrm{~N} . \mathrm{S})$ & $>0.05(\mathrm{~N} . \mathrm{S})$ \\
\hline
\end{tabular}

N.S- Not significant Correlation 


\section{Discussion}

The process of invasion and metastasis is of paramount clinical importance, but the basic mechanism underlying these events are largely unknown and not clearly understood. The metastatic carcinoma cell has been linked to a decathlon champion because of the multiple properties it needs to have to create metastatic deposits. ${ }^{14}$

The initial local steps in the metastatic process include expansion of the primary tumor, invasion of host tissue with disruption of the basement membrane, digestion (proteolysis) of the extra cellular matrix, and invasion of lymphatic vessels, blood vessels, or both. ${ }^{15}$

Metastasis is a multi step process in which tumor cells gain access to the vasculature in the primary tumor, survive in the circulation, arrest in the microvasculature of the target organ, exit from this microvasculature, and proliferate into the target tissues. Tumor cells rarely shed into the circulation before the primary tumor is vascularized, and micro metastases cannot grow to a detectable size until after they have become vascularized. Thus angiogenesis is necessary at the beginning as well as at the end of metastatic cascade. It is likely that a primary tumor containing a high proportion of angiogenic tumor cells called metastatic clones, will generate metastases that are already angiogenic when they begin their growth in the target organ. ${ }^{16}$

Neo vascularization permits the shedding of cells from the primary tumor, and there by promote metastasis; on the contrary decreased angiogenesis is associated with a decreased rate of metastasis. ${ }^{17}$

The angiogenic activity is mediated by specific angiogenic molecules released from the tumor cells per se or from cells recruited to the area (i.e, macrophages and mast cells). It is now known that, in solid tumors, there is a prevascular phase during which little or no angiogenic factors are released by the cells, despite their proliferative capacity, such tumor cells cannot expand the tumor population beyond a few cubic milli meters. Thus the absence of the neovascularization severely restricts the size of tumor mass. But when it is sufficient to induce neo vascularization, the onset of angiogenic activity permits rapid expansion of the tumor.

The onset of angiogenic activity does not require that all the tumor cells become angiogenic. In fact, "tumor appear to be heterogenous for angiogenic activity" Consequently, it is imperative to determine micro vessel density in the areas of the most intense neovascularity. The highly angiogenic tumor cells are the likely source of growing metastatic foci. Also, an increase in the propotion of angiogenic tumor cells within a primary tumor is one mechanism by which overall neovascularization could increase. Indeed, these breast carcinomas from the current study showing the highest micro vessel counts likely contained higher percentage of angiogenic cells than the tumor with the low counts. Moreover, this mechanism provides an explanation for the strong association of increasing neo vascularization with increased risk of metastasis. ${ }^{16}$

Tumor angiogenesis can facilitate metastatic spread in some ways. For example, newly proliferating capillaries have fragmented basement membranes and are leaky, making them more accessible to tumor cells than mature vessels. In addition, the invasive chemotactic behavior of endothelial cells at the tips of growing capillaries is facilitated by their secretion of collagenases and plasminogen activator. These degradative enzymes may also facilitate the escape of tumor cells into the tumor neovasculature. And, it has been shown that greater numbers of tumor vessels increase the opportunity for tumor cells to enter the circulation.

However, it must be emphasized that neovascularization permits, but does not guarantee, progressive tumor spread. For example typical pulmonary carcinoid tumors are highly vascular but rarely metastasize to distant sites. Thus an angiogenic metastatic cell must overcome many other obstacles to become a successful metastasis. ${ }^{16}$

Metastases in breast cancer results from the interaction of several factors, including angiogenesis, tissue invasion, adhesion molecules (cell/cell and cell/matrix adhesion), and regulation of cell movement.

High expression of CD 31 has been correlated with poor out come and aggressive histological features. All these data suggest that PECAM-1/CD 31 expression in tumors may also have role in the development of metastasis. In platelets, the cytoplasmic domain of PECAM-1 becomes specifically associated with the contractile elements of cyto skeleton that are involved in the platelet spreading process.

In endothelial cell culture PECAM-1 is diffusely distributed on the cell surface until cell to cell contact is achieved at which point PECAM-1 becomes localized to intercellular junctions. In tissue sections from breast cancer, samples of the PECAM-1 staining is observed along cell membranes of endothelial cells of capillaries and venules.

From our observations it can be speculated that the activation of PECAM-1 in cancer that results in its sub cellular distribution in endothelium plays a role in the metastatic disease by facilitation tumor cell adhesion to blood vessel walls. ${ }^{10}$

Angiogenesis was assessed by immune detection of the endothelial cell marker-CD 31 which is more specific for the vasculature than other endothelial markers like ulex europaeus agglutinin lectin, F-VIII or CD-34. Ulex europaeus agglutinin lectin stains normal endothelial cells but also a wide variety of other cells and particularly connective tissue cells. Factor -VIII is partially absent from the capillary endothelium in tumor tissue and like CD-34, F- VIII also displays lymphatic immune staining. ${ }^{17,18}$ 
Weidner et al in 1991 has showed a significant correlation between the microvessel density in histological sections of invasive breast carcinoma and the occurrence of local or distant metastases. ${ }^{12}$

Folk man et al in 1992 has showed that a micro vessel count in the area of most intense neovascularization in breast carcinoma is both an independent and highly significant prognostic factor in predicting overall survival and relapse- free survival in all patients, including the node positive subset, and even more importantly the node negative subset. Thus determination of micro vessel density in invasive breast carcinoma could be valuable in selecting the nodenegative patients who are at high risk for having occult metastasis at presentation and thus are possible candidates for systemic adjuvant therapy. ${ }^{16}$

Bosari et al in 1992 has done micro vessel quantitation and they showed correlation in micro vessel density with lymph node status, recurrence rate, tumor size. ${ }^{13}$

The results of their investigation confirm the association of high vessels counts in invasive breast carcinoma with the presence of axillary lymph node metastasis, which is consistent with the role of angiogenesis in the metastatic process..$^{12,13,16}$

In addition significant difference was found in micro vessel density between patients with nodenegative carcinoma in whom disease recurred at a later stage and patients with long term disease free survival. Thus it appears that a higher number of vessels may also play a role in the long term aggressiveness of tumor. ${ }^{16}$

In their study no association was found between micro vessel density and tumor size. ${ }^{13}$

Horak et al in 1992 has done microvessel quantititation by staining their endothelial cells immunocyto chemically for factor VIII and (PECAM) CD 31. They proved monoclonal anti body to PECAM is more sensitive than antibodies to factor VIII antigen. ${ }^{11}$

Colette Charpin et al in1995 has done CD 31 quantitative immunocyto chemical assays in invasive breast carcinoma and correlated with the Nottingham prognostic index, tumor size, node status and tumor type. They got significant correlation with Nottingham prognostic index but not with the tumor size. ${ }^{10}$

In our study we also had chosen CD-31 for microvessel staining for endothelial staining of blood vessels.

Goulding et al in 1995, has showed association between angiogenesis and traditional prognostic indicators such as grade, tumor type and lymph node status but no association between micro vessels count and tumor size, estrogen receptor status. ${ }^{19}$

Knopp et al in 1999 has done micro vessel quantitation in breast tumors by MRI contrast enhancement by selecting hot spot areas and compared tumor types.
They conformed Infiltrating Duct cell Carcinoma is more frequently associated with axillary node metastases and had higher micro vascular density.

Infiltrating lobular carcinoma is characterized by diffuse infiltrative growth and low micro vascular density. ${ }^{20}$

In our study the microvessel density was higher in stage II and III lymph node status and Infiltrating duct cell carcinomas, also found high vascular density in histological grade II and grade III. Our findings correlates with knopp et al and other related studies.

In recently published most of the articles. ${ }^{10,11,16,19}$ shows association between microvessel density and tumor grading, lymph node status, and tumor type. Our findings are similar to their findings with definitive significance (all 'p' value <0.05)

But tumor size has poor or no correlation with microvessel density, as seen in Bosari et al study and Goulding et al study. ${ }^{13,19}$ Our finding matches with their finding.

\section{References}

1. Carmeliet P: Manipulating angiogenesis in medicine. J Intern Med 2004;255:538.

2. Brown JM, Giaccia Aj (1998). The unique physiology of solid tumors: opportunities (and problems) of cancer therapy. Cancer Research 58(7):pp.1408-16.PMID 9537241.

3. Bergers G, Benjaman LE(2003). Tumor angiogenesis and the angiogenic switch. Nature Reviews. Cancer 3(6):pp.401-10.PMID:12778130.

4. Rafii S, Heissig B, Hattori K (2002). Efficient Mobilization and recruitment of marrow derived endothelial and haemopoietic stem cells by adenoviral vectors expressing angiogenic factors. Gene therapy 9(10):pp.631-41.PMID120320709.

5. McDougall, S.R, Anderson, A.R.A, Chaplain, M.A.J. Mathemetical modeling of dynamic adaptive tumorinduced angiogenesis: Clinical implications and therapeutic targeting strategies. Journal of Theoretical biology, pp.241.2006 (cites Folkman,1971).

6. W. Jeffrey Allard, Jeri Matera, M. Craig Miller et al. (2004). Tumor cells circulate in the peripheral blood of All Major carcinomas but not in healthy subjects of patients with non-malignant diseases. Clin Cancer Research 10(20):pp.6897-6904.

7. Enzyme eliminated by cancer cells holds promise for cancer treatment.

Link:www.eurekalert.org/pub_releases/2007-07/mcogeeb071807.php. (accessed on $3^{\text {rd }}$ Nov2013).

8. Noel Weidner (1995). Intratumor microvessel density as a prognostic factor in cancer. Americal Journal of Pathology.Vol.147, No.1:pp. 9-16.

9. Juan Rosai, M.D, Prognosis; Breast; chapter 20; Rosai and Ackerman's Surgical pathology; Edn VIII. p. 1624. Mosby publishers;Elsevierhealth.

10. Colette Charpin, M.D, ${ }^{1}$ Eenedicte Devictor, $\mathrm{PhD}$, Denise Bergeret, $\mathrm{PhD}, 2$ et al ; CD 31 Quantitative

Immunocytochemical assays in Breast Carcinomas Correlation with Current Prognostic Factors; Anatomic Pathology. American Journal of Clinical Pathology; Vol. 103; no.4; April 1995; pp.443-448. 
11. Elizabeth R. Horak, Russel Leek, Nora Klenr, Susan Lejeune et al, Angiogenesis assessed by platelet/ endothelial cell adhesion molecule antibodies, as indicator of node metastasis and survival in breast cancer. The Lancet, Vol: 340; Nov 7, 1992, pp. 1120-1124.

12. Noel, Weidner, MD, Joseph P. Semple, M.D., William Ret al. Tumors Angiogenesis and Metastasis - Correlation in Invasive Breast Carcinoma. The New England Journal of Medicine; Vol. 324, No.1, January 3;1991;pp.1-7.

13. Silvano Bosari, MD, Arthur K.C. Lee, MBBS, Ronald A. Delellis, MD et al, Microvessel Quantization and Prognosis in invasive Breast Carcinoma. Human Pathology. Volume 23, No. 7, July 1992, pp. 755-761.

14. Sugar Baker EV: Cancer Metastasis: A product of tumor - host Interactions. Current Problems in cancer 3 , 1979:pp. 1-59.

15. Liotta LA, Steeg PS, Stetler-Stevenson WG: Cancer Metastasis and angiogenesis; An Imbalance of positive and negative regulations. Cell 64, 1991: pp. 327- 336.

16. Judah Folkman, Franco Pozza, Pierantonio et al, Tumor angiogenesis: A new significant and dependent Prognostic Indicator in early stage breast carcinoma. Journal of the National Cancer Institute Vol. 84, No. 24, December 16, 1992, pp. 1875-1887.

17. D.V Parums, JL Cordell, K Micklem, A.R. Heryet, K.C Gatter et al, Jc 70: a new monoclonal antibody that detects vascular endothelium associated antigen on routinely processed tissue sections. Journal of Clinical pathology; 1990, Vol. 43;P. No. 752-757.

18. Efthymia Tsoli, BSc Mphil, Panayotis Zacharatos, BSc, $\mathrm{PhD}$ et al, Growth Index is Independent of Micro vessel Density in Non- Small- cell Lung carcinomas. Human Pathology; vol. 33, No. 8(August 2002), pp. 812-818.

19. H. Goulding, N.F. Nik Abdul Rashid, J.F. Robertson et al. Assessment of Angiogenesis in Breast carcinoma; An important factor in prognosis? ; Human pathology, Vol: 26 No: 11 (November 1995). (pp. 1196- 1200).

20. M.V Knopp, MD, ${ }^{1 *}$ E.Weiss, ${ }^{1}$ H.P.Sinn, MD,${ }^{2}$ J. Mattern et al. Patho physiologic basis of contrast enhancement in breast tumors. Journal of Magnetic Resonance Imaging 10: pp.260-266(1999). 